\title{
The benefit of taxane-based therapies over fluoropyrimidine plus platinum (FP) in the treatment of esophageal cancer: a meta-analysis of clinical studies
}

This article was published in the following Dove Medical Press journal:

Drug Design, Development and Therapy

\begin{abstract}
Tao Wang ${ }^{1, *}$
Jie $\mathrm{Yu}^{1, *}$

Min Liul,*

Yanliang Chen'

Caiyun Zhu'

Lin $\mathrm{Lu}^{2}$

Mingzhu Wang ${ }^{2}$

Lingfeng $\mathrm{Min}^{3}$

Xinxin $\mathrm{Liu}^{4}$

Xizhi Zhang'

Johannes A Gubat ${ }^{5}$

Yong Chen'

'Department of Medical Oncology, Clinical Medical College, Yangzhou University, Yangzhou, Jiangsu, China; 2Department of Medical Oncology, Dalian Medical University, Dalian, Liaoning, China; ${ }^{3}$ Department of Respiratory Medicine, Clinical Medical College, Yangzhou University, Yangzhou, Jiangsu, China; ${ }^{4}$ Department of Gastrointestinal Surgery, Clinical Medical College, Yangzhou University, Yangzhou, Jiangsu, China; ${ }^{5}$ Division of Oncology and Pathology, Department of Clinical Sciences, Lund University, Lund, Sweden

*These authors contributed equally to this work
\end{abstract}

Correspondence: Yong Chen Department of Medical Oncology, Clinical Medical College, Yangzhou University, No 98 Nantong West Road, Guangling District, Yangzhou, Jiangsu, China

Tel +865I487373825

Email chenyong_jsyz@sina.com
Purpose: Fluoropyrimidine plus platinum (FP) is currently the standard treatment for esophageal cancer (EC). In recent years, taxane-based chemotherapy has also been used and has shown good efficacy in EC. This study aims to investigate the advantages of taxane-based over FP chemotherapy, as well as discuss its drawbacks, in the treatment of EC.

Patients and methods: A literature search was done for studies comparing clinical outcomes between taxane-based and FP chemotherapy in EC. Pooled analyses were performed to compare the efficacy and grade 3/4 adverse events in patients who received neoadjuvant chemotherapy (NACT), neoadjuvant chemoradiotherapy (NACRT), or definitive chemoradiotherapy (dCRT). Subgroup analyses were also conducted in esophageal squamous cell carcinoma (ESCC).

Results: Thirty-one studies with a total of 3,912 patients were included in the analysis. Better long-term survival was found in patients who received taxane-based NACT (progression-free survival (PFS): pooled $H R=0.58, P=0.0008$; and overall survival $(\mathrm{OS})$ : pooled $H R=0.50$, $P<0.00001$ ) and dCRT (PFS: pooled HR=0.75, $P<0.0001$ ). In NACRT, taxane-based treatment and FP showed similar efficacy. In ESCC patients, taxane-based treatment showed better OS (NACT: pooled HR=0.57, $P=0.02$; NACRT: pooled HR=0.51, $P=0.03$; and dCRT: pooled $\mathrm{HR}=0.73, P<0.0001)$ than FP chemotherapy. Furthermore, taxane-based therapy also showed a better short-term response (complete response (CR), objective response rate (ORR), disease control rate (DCR), or pathologic complete response (pCR). However, taxane-based therapy was significantly correlated with a higher incidence of grade 3/4 leukopenia, neutropenia, and diarrhea. Conclusion: Compared to FP, taxane-based therapy produced better clinical response and outcomes in EC patients receiving NACT or dCRT, and in all types of therapy in patients with ESCC. Taxane-based treatment is associated with more frequent toxicity.

Keywords: digestive cancer, chemotherapy, survival, clinical cancer research

\section{Introduction}

Esophageal cancer (EC) is one of the most common malignancies worldwide, especially in developing countries. ${ }^{1}$ From 2012 to 2015, it was estimated that the worldwide incidence and mortality of EC increased from 455,800-483,000 and from 400,200-439,000 respectively. ${ }^{1,2}$ In China, EC is estimated as the third most common cancer and the fourth leading cause of cancer death among all the cancer types in $2015 .^{3}$ EC is usually diagnosed at an advanced stage as it is clinically inconspicuous and is characterized by high rates of locoregional recurrence and distant metastasis after primary surgical treatment. ${ }^{4}$ 
The treatment options for EC include surgery with or without neoadjuvant treatment (chemotherapy or chemoradiotherapy $)^{5}$ and definitive chemoradiotherapy (dCRT). ${ }^{6}$ Therefore, the choice of treatment plays a very important role in the prognosis of EC. Preoperative chemotherapy or chemoradiotherapy has become a treatment of choice for most locally advanced resectable cases, ${ }^{5,7}$ and the definitive concurrent chemoradiotherapy (CCRT) has been established as a standard treatment for unresectable, locally advanced cases since results from the RTOG 85-01 trial were reported. ${ }^{6}$ During the last decades, the overall prognosis of EC has slowly improved, partly because of the increasing practice of multidisciplinary management. Despite this, the 5-year survival rate remains low, with only about $19 \%$ based on the US National Cancer Institute's report in $2018 .^{8}$

In the clinic, the most commonly used regimens are those consisting of platinum (carboplatin/cisplatin) combined with either fluorouracil (5-FU) or taxanes (paclitaxel/docetaxel) and their modifications. Fluoropyrimidine plus platinum (FP), especially cisplatin plus 5-FU (CF), was mostly used as a first-line treatment for several years, and became a standard regimen and category one recommendation for EC in many countries. ${ }^{9,10}$ At the same time, taxane-based chemotherapy or chemoradiotherapy as a first-line therapy had also been shown to be effective in EC. ${ }^{4,11,12}$ As a result, more and more studies attempted to find clinical benefits of taxane-based therapy over the FP regimen. Some studies indicated that taxane-based regimens were more effective than FP, ${ }^{11,13-20}$ while other studies showed lower efficacy ${ }^{21-23}$ or higher toxicity. ${ }^{24-26}$ In view of these controversial results, we made this meta-analysis to investigate the benefits and disadvantages of taxane-based first-line therapy compared with FP therapy in the treatment of EC.

\section{Materials and methods}

\section{Search strategy and study selection}

Medline and Embase were searched for publications up to September 2017. The following search terms in the title were used without any language restriction: (esophageal OR esophagus OR oesophageal OR esophagus) AND (tumor OR cancer OR carcinoma OR neoplasm OR neoplasms) and (docetaxel OR paclitaxel OR taxane). In addition, references in all relative researches were reviewed for any further eligible studies. All studies included in the meta-analysis should meet the following criteria: 1) be a randomized controlled trial (RCT) or cohort study and 2) investigate curative effects or adverse events between taxane-based regimens and FP in EC. If more than one publication reported results from the same study, the latest updated data was extracted. Studies that included recurrent or metastatic EC and any prior interventions except for diagnostic biopsy were excluded from the analysis.

\section{Endpoints of interest}

Based on the chemotherapy regimens used, patients were classified into two main groups, namely the taxane group and the FP group. Patients were further divided into two subgroups, namely the neoadjuvant therapy (NAT) group and the dCRT group, according to the subsequent surgical intervention. The primary outcome measures were complete response (CR), objective response rate (ORR), disease control rate (DCR); hazard ratios (HRs) with their $95 \%$ CIs for progression-free survival (PFS) and overall survival (OS) after treatment; and grade 3/4 adverse events (anemia, neutropenia, leukopenia, thrombocytopenia, anorexia, nausea, vomiting, diarrhea, esophagitis, and pneumonia). Pathologic complete response (pCR) and $\mathrm{R} 0$ resection in the NAT group were also noted.

\section{Data extraction}

All studies searched were reviewed by two authors ( $T$ Wang and M Liu) independently to exclude irrelevant or duplicate publications. Data were extracted from all included studies. In the event of a discrepancy, a third reviewer (Y Chen) reviewed the study in question to reach a consensus. Details extracted from the included studies were the name of the first author, year of publication, study period, geographic area, sample size, median age, median follow-up, chemotherapy regimen, median radiation dose, treatment strategy, pathological type, clinical stage, and research type (Table 1). Odds ratio (OR) and its $95 \% \mathrm{CI}$ were used to express the frequencies of $\mathrm{CR}, \mathrm{ORR}, \mathrm{DCR}, \mathrm{pCR}, \mathrm{R} 0$ resection, and different kinds of adverse events. HRs and their $95 \% \mathrm{CI}$ for PFS and OS were also extracted from different studies. If the data for HR and its $95 \%$ CI cannot be acquired, the methods outlined by Tierney et $\mathrm{al}^{27}$ were applied to get an estimated value. From Kaplan-Meier curves, Engauge Digitizer version 4.1 (available from http://digitizer.sourceforge.net/) was used to extract data which were then put in the calculation spreadsheet appended to Tierney et al's paper. Besides, data for disease-free survival (DFS) and recurrence-free survival (RFS) were all defined as PFS. Furthermore, patients who received sequential CRT were also included. 


\section{Quality assessment and statistical analysis}

The quality of cohort studies was assessed using the nine-star Newcastle-Ottawa Quality Assessment Scale (http://www. ohri.ca/programs/clinical epidemiology/oxford.asp), while the Cochrane risk of bias tool was used for RCTs. In the ninestar Newcastle-Ottawa Quality Assessment Scale, scores of $1-3,4-6$, and 7-9 means low quality, medium quality, and high quality, respectively.

Studies with a median follow-up period $<2$ years were excluded when survival was analyzed. Pooled HRs for OS and PFS and pooled ORs for pCR, R0 resection, CR, ORR, DCR, and adverse events were obtained using the RevMan 5.3 analysis software. At the same time, a Z-test was used to examine the statistical significance of pooled estimates and the statistical heterogeneity was assessed by $\mathrm{I}^{2}$ tests. Whenever there was significant heterogeneity $(P<0.05$ or $\mathrm{I}^{2}>50 \%$ ), the random effect model was applied to analyze the estimated values. Otherwise, the fixed effect model was used. ${ }^{28}$ Tests for funnel plot asymmetry were used to examine bias in the results of meta-analyses. Begg's and Egger's tests, which were performed using the software of STATA (version 14.0; StataCorp, College Station, TX, USA), were used to estimate the publication bias of analysis involving at least 10 studies, and $P<0.05$ was considered to be of statistical significance.

\section{Results}

\section{Search results and description of studies}

A total of 516 potentially relevant articles were identified from the Medline and Embase databases. There were 462 articles excluded through examining the titles, abstracts, and full texts. In the remaining 54 articles, 24 were further excluded because they were duplicates (seven articles), had patients with prior intervention (five studies), did not have data that could be extracted (seven studies), or the non-taxanebased regimen was not FP (five studies). Through reviewing the references of the remaining 30 articles, two additional relevant studies were found and added. Finally, 32 articles with 3,912 patients were included in the final meta-analysis. A total of 26 articles $^{11-20,24-26,29-41}$ were published in full text, while six articles ${ }^{21-23,42-44}$ were only in abstract form. Two of the 32 articles $^{14,37}$ described one study. Hence, there were 31 studies altogether (seven RCTs and 24 cohort studies). The detailed steps of study selection were summarized in Figure 1.

There were 18 studies from Asian countries (12 from China, six from Japan) and 13 studies from Western countries (six from America, three from the Netherlands, two from
Canada, one from Germany, and one from the Czech Republic). Among all the included studies, 17 analyzed the benefits of neoadjuvant taxane-based therapy (neoadjuvant chemotherapy, NACT: seven studies; neoadjuvant chemoradiotherapy, NACRT: 10 studies), 11 studies analyzed the clinical benefits of taxane-based dCRT, and three studies analyzed the benefits of both dCRT and NACRT in EC. Taxane-based regimens included taxane-based monotherapy (paclitaxel/docetaxel), two-drugs, or three-drugs therapy. The radiation doses for dCRT and NACRT ranged from 36-70 Gy and 36-69 Gy, respectively. HRs for OS and PFS could be directly or indirectly acquired on the basis of Kaplan-Meier curves from 20 studies and 13 studies, respectively. Most of the studies reported relevant adverse reactions and complications, and the common adverse events were anemia, leukopenia, neutropenia, thrombocytopenia, anorexia, nausea, vomiting, diarrhea, esophagitis, and pneumonia. The characteristics of all included studies published from 2000-2017 are shown in Table 1.

For patients with resectable or potentially resectable EC, NAT tends to be a good option, ${ }^{5,7}$ while dCRT is usually administrated in unresectable cases. In this light, we conducted subgroup analyses of NACT, NACRT, and dCRT.

\section{Quality assessment and publication bias}

The quality scores of included cohort studies are summarized in Table S1 and Figure S1A, which ranged from 6-9, with a median score of 7. All these included studies had medium-to-high quality. No high risk of bias was found in any RCTs (Figures S1B and C). Funnel plots, Begg's, and Egger's tests were used to assess publication bias, and no publication biases were found. Plots of Begg's and Egger's tests are shown in Figure S2.

\section{Taxane-based therapy confers better disease control and long-term survival but causes severe toxicity more frequently in patients compared with FP in patients who received NACT}

Eight studies reported taxane-based NACT in EC, and seven were comparisons of TPF (taxane+platinum+ fluoropyrimidine) and FP, while one was a comparison of paclitaxel+carboplatin and FP. From these clinical studies, we found that short-term clinical responses except CR were significantly better in patients who received taxane-based therapy than in those who received FP therapy 
Table I The characteristics of all included studies

\begin{tabular}{|c|c|c|c|c|c|c|}
\hline Author & Year & $\begin{array}{l}\text { Study } \\
\text { period }\end{array}$ & $\begin{array}{l}\text { Geographic } \\
\text { area }\end{array}$ & $\begin{array}{l}\text { Sample size } \\
\text { (taxane/ } \\
\text { non-taxane) }\end{array}$ & $\begin{array}{l}\text { Median age } \\
\text { (taxane/non- } \\
\text { taxane, years) }\end{array}$ & $\begin{array}{l}\text { Median follow-up } \\
\text { (taxane/non-taxane, } \\
\text { months) }\end{array}$ \\
\hline Adelstein et al & 2000 & |991.08-1997.07 & America & $112(40 / 72)$ & 60 & 33 \\
\hline Roof et al & 2006 & 1994-2002 & America & I $64(83 / 8 I)$ & $61 / 64$ & 54 \\
\hline Bader et al & 2008 & $1993-2000$ & German & $67(35 / 32)$ & $51.6 / 56.4$ & $78.3 / 109.3$ \\
\hline Hsu et al & 2008 & 1999-2004 & China & $127(57 / 70)$ & $57 / 62.3$ & 35 \\
\hline Zemanova et al & 2010 & $2001.01-2005.08$ & Czech Republic & $107(44 / 63)$ & $58 / 60$ & 52 \\
\hline Courrech Staal et al & 2011 & 1997-2007 & Netherlands & $81(16 / 65)$ & $70 / 60$ & 15 \\
\hline Chen et al & 2011 & $2005.01-2007.06$ & China & $48(24 / 24)$ & 57 & - \\
\hline Wu et al & 2012 & $2008.07-2009.12$ & China & $154(77 / 77)$ & $61 / 60$ & - \\
\hline Zhao et al & 2012 & $2005.01 .01-2008.05 .3 \mathrm{I}$ & China & $90(45 / 45)$ & - & 14.2 \\
\hline Bai et al & 2013 & $2009.08-2011.05$ & China & $74(36 / 38)$ & $54 / 56$ & - \\
\hline Blom et al & 2014 & $2005.01-2010.07$ & Netherlands & $165(92 / 73)$ & $62 / 64$ & - \\
\hline Honing et al & 2014 & $1996-2008$ & Netherlands & $102(55 / 47)$ & $64.8 / 62.5$ & - \\
\hline Katada et al & 2014 & $2007.09-2010.12$ & Japan & $79(38 / 4 I)$ & $64.4 / 64.2$ & $27 / 42$ \\
\hline Schellenberg et al & 2014 & $20|0.02-20| 3.02$ & Canada & $112(39 / 73)$ & - & - \\
\hline Thomay et al & 2014 & $2008.0 \mathrm{I}-20 \mathrm{I} 3.06$ & America & 7I (33/38) & - & $9.1 / 18.2$ \\
\hline Berman et al & 2014 & $2008.07-2013.10$ & America & $100(49 / 51)$ & 65 & - \\
\hline Kushida et al & 2014 & $200 \mathrm{I}-2007$ & Japan & $95(55 / 40)$ & $61.9 / 60.4$ & - \\
\hline Yang et al & 2015 & $2008.03-2010.01$ & China & $68(34 / 34)$ & $59 / 56$ & 9 \\
\hline Wang et al & 2015 & $2012.06-2014.10$ & China & $53(25 / 28)$ & - & - \\
\hline Nomura et al & 2015 & $2003.01-2013.01$ & Japan & $209(60 / 149)$ & $61 / 62$ & 32.4 \\
\hline Ui et al & 2015 & $2007.04-2011.09$ & Japan & $76(38 / 38)$ & $62 / 66$ & 22.8 \\
\hline Boggs et al & 2015 & $1992-2012$ & America & $159(30 / 129)$ & - & - \\
\hline Sun et al & 2016 & 2009.03-2014.1I & China & $179(83 / 96)$ & $61 / 59$ & 28 \\
\hline Hu et al & 2016 & $2009.01-2013.12$ & China & $202(105 / 97)$ & $61.3 / 61.1$ & 44.6 \\
\hline Zhang et al & 2016 & $2002-2013$ & China & $317(161 / 156)$ & $58 / 56.4$ & $21 / 24$ \\
\hline Yamashita et al & 2016 & $2007.09-2012.08$ & Japan & $79(38 / 4 I)$ & - & 49 \\
\hline
\end{tabular}




\begin{tabular}{|c|c|c|c|c|c|c|c|}
\hline $\begin{array}{l}\text { Chemotherapy } \\
\text { regimens }\end{array}$ & $\begin{array}{l}\text { Median radiation } \\
\text { dose (taxane/non- } \\
\text { taxane, Gy) }\end{array}$ & $\begin{array}{l}\text { Treatment } \\
\text { strategy }\end{array}$ & \begin{tabular}{|l|} 
Pathological \\
types
\end{tabular} & $\begin{array}{l}\text { Clinical } \\
\text { stage }\end{array}$ & $\begin{array}{l}\text { Research } \\
\text { type }\end{array}$ & Quality & Reference \\
\hline $\begin{array}{l}\text { PTX+DDP vs } \\
5-F U+D D P\end{array}$ & 69 & NACRT & AC/SCC/other & II-IV/unknown & CS & 7 & 26 \\
\hline $\begin{array}{l}\mathrm{PTX}+5-\mathrm{FU}+\mathrm{DDP} \text { vs } \\
5-\mathrm{FU}+\mathrm{DDP}\end{array}$ & 58.5 & NACRT & $\mathrm{AC} / \mathrm{SCC}$ & II, III, IV & CS & 7 & 31 \\
\hline $\begin{array}{l}P T X+5-F U+L V+D D P \\
\text { vs } 5-F U+L V+D D P\end{array}$ & - & NACT & $A C$ & $\mathrm{~T} 3 / 4$ & CS & 7 & 33 \\
\hline $\begin{array}{l}\text { PTX+DDP vs } \\
\text { 5-FU+DDP }\end{array}$ & $36 / 60$ & dCRT/NACRT & ScC & II, III & CS & 7 & 32 \\
\hline $\begin{array}{l}\mathrm{PTX}+5-\mathrm{FU}+\mathrm{CBP} / \mathrm{DDP} \\
\text { vs } 5-\mathrm{FU}+\mathrm{CBP} / \mathrm{DDP}\end{array}$ & 45.1 & NACRT & AC/SCC/other & II-IVa & CS & 7 & 25 \\
\hline $\begin{array}{l}\text { PTX+CBP vs } \\
5-F U+D D P\end{array}$ & $50.4 / 36-50$ & CCRT/NACRT & AC/SCC/AC-SCC & II-IVa & CS & 7 & 30 \\
\hline $\begin{array}{l}\text { DTX+DDP vs } \\
5-F U+D D P\end{array}$ & 60 & CCRT & SCC & III, IVa & RCT & - & 34 \\
\hline $\begin{array}{l}\text { DTX+DDP vs } \\
5-F U+D D P\end{array}$ & 40 & NACRT & SCC & T3NO-IMO & RCT & - & 40 \\
\hline $\begin{array}{l}\text { DTX+DDP vs } \\
5-F U+D D P\end{array}$ & 50.4 & CCRT & SCC & II-IVa & RCT & - & 20 \\
\hline $\begin{array}{l}\text { DTX+DDP vs } \\
5-F U+D D P\end{array}$ & 60 & CCRT & ScC & IIB-IIIB & RCT & - & 35 \\
\hline $\begin{array}{l}\text { PTX+CBP vs } \\
5-\mathrm{FU}+\mathrm{DDP}\end{array}$ & $41.4 / 50.4$ & NACRT & AC/SCC/other & TI-3NO-IMO & CS & 7 & 29 \\
\hline $\begin{array}{l}\text { PTX+CBP vs } \\
5-F U+D D P\end{array}$ & 50.4 & CCRT & $\mathrm{AC} / \mathrm{SCC}$ & I-IV & CS & 7 & 11 \\
\hline $\begin{array}{l}\mathrm{DTX}+5-\mathrm{FU}+\mathrm{DDP} \text { vs } \\
5-\mathrm{FU}+\mathrm{DDP}\end{array}$ & - & NACT & SCC & II, III & CS & 7 & 37 \\
\hline $\begin{array}{l}\text { PTX+CBP vs } \\
5-\text { FU+DDP }\end{array}$ & 50 & NACRT & - & - & CS & 7 & 42 \\
\hline $\begin{array}{l}\text { PTX+CBP vs } \\
5-F U+D D P\end{array}$ & 50.4 & NACRT & - & - & CS & 9 & 23 \\
\hline $\begin{array}{l}\mathrm{PTX}+\mathrm{CBP} \text { vs } \\
\text { 5-FU+platinum }\end{array}$ & 50.4 & NACRT & $\mathrm{AC} / \mathrm{SCC}$ & II, III, IV & CS & 6 & 43 \\
\hline DTX vs 5-FU+DDP & 40 & NACRT & Scc & T2-4NI-3M0 & CS & 7 & 36 \\
\hline $\begin{array}{l}\text { PTX+LBP vs } \\
\text { 5-FU+DDPP }\end{array}$ & $60-70$ & CCRT & SCC & III, IVa & RCT & - & 17 \\
\hline $\begin{array}{l}\text { PTX+DDP vs } \\
\text { 5-FU+DDP }\end{array}$ & $56-60$ & CCRT & $\mathrm{AC} / \mathrm{SCC}$ & - & RCT & - & 38 \\
\hline $\begin{array}{l}\text { DTX+5-FU+DDP vs } \\
\text { 5-FU+DDP }\end{array}$ & - & NACT & SCC & II, III & $C S$ & 7 & 18 \\
\hline $\begin{array}{l}\mathrm{DTX}+5-\mathrm{FU}+\mathrm{DDP} \text { vs } \\
5-\mathrm{FU}+\mathrm{DDP}\end{array}$ & - & NACT & $\mathrm{AC} / \mathrm{SCC}$ & II-IV & CS & 7 & 19 \\
\hline $\begin{array}{l}\text { PTX+CBP/DDP vs } \\
5-\mathrm{FU}+\mathrm{DDP}\end{array}$ & 50.4 & NACRT & $\mathrm{AC} / \mathrm{SCC}$ & II, III, IV & CS & 6 & 39 \\
\hline $\begin{array}{l}\text { PTX/DTX+CBP/ } \\
\text { DDP/NDP vs } \\
\text { fluoropyrimidine+ } \\
\text { DDP/NDP }\end{array}$ & 56 & dCRT & SCC & II-IV & CS & 7 & 12 \\
\hline $\begin{array}{l}\text { PTX+DDP vs } \\
5-F U+D D P\end{array}$ & $54-60$ & CCRT & SCC & IIB, III & CS & 7 & 13 \\
\hline $\begin{array}{l}\text { DTX+DDP vs } \\
5-\text { FU+DDP }\end{array}$ & $50-70$ & CCRT & SCC & II-IVa & CS & 9 & 16 \\
\hline $\begin{array}{l}\text { DTX+5-FU+DDP vs } \\
5-F U+D D P\end{array}$ & - & NACT & SCC & II, III & CS & 7 & 14 \\
\hline
\end{tabular}


Table I (Continued)

\begin{tabular}{|c|c|c|c|c|c|c|}
\hline Author & Year & $\begin{array}{l}\text { Study } \\
\text { period }\end{array}$ & $\begin{array}{l}\text { Geographic } \\
\text { area }\end{array}$ & $\begin{array}{l}\text { Sample size } \\
\text { (taxanel } \\
\text { non-taxane) }\end{array}$ & $\begin{array}{l}\text { Median age } \\
\text { (taxane/non- } \\
\text { taxane, years) }\end{array}$ & $\begin{array}{l}\text { Median follow-up } \\
\text { (taxane/non-taxane, } \\
\text { months) }\end{array}$ \\
\hline Ojima et al & 2016 & $2008.01-2012.12$ & Japan & $77(48 / 29)$ & $65 / 68$ & $37 / 48$ \\
\hline Haisley et al & 2016 & $2000.01-2015.07$ & America & I 42 (87/55) & - & - \\
\hline Fang et al & 2017 & $2009.01-2013.12$ & China & $82(4 I / 4 I)$ & - & 28.4 \\
\hline Akiyama et al & 2017 & $2007.03-2016.12$ & Japan & $63(29 / 34)$ & $64 / 64.3$ & - \\
\hline Chen et al & 2017 & $2012.04-2015.07$ & China & $436(218 / 218)$ & - & - \\
\hline Sim et al & 2017 & $2011-2015$ & Canada & $101(40 / 61)$ & 62 & 43 \\
\hline
\end{tabular}

Abbreviations: 5-FU, fluorouracil; AC, adenocarcinoma; ADM, adriamycin; CBP, carboplatin; CCRT, concurrent chemoradiotherapy; CPT-II, irinotecan; CS, cohort study; dCRT, definitive chemoradiotherapy; DDP, cisplatin; DTX, docetaxel; LBP, lobaplatin; NACRT, neoadjuvant chemoradiotherapy; NACT, neoadjuvant chemotherapy; NAT, neoadjuvant therapy; NDP, nedaplatin; PTX, paclitaxel; RCT, randomized controlled trail; SCC, squamous cell carcinoma.

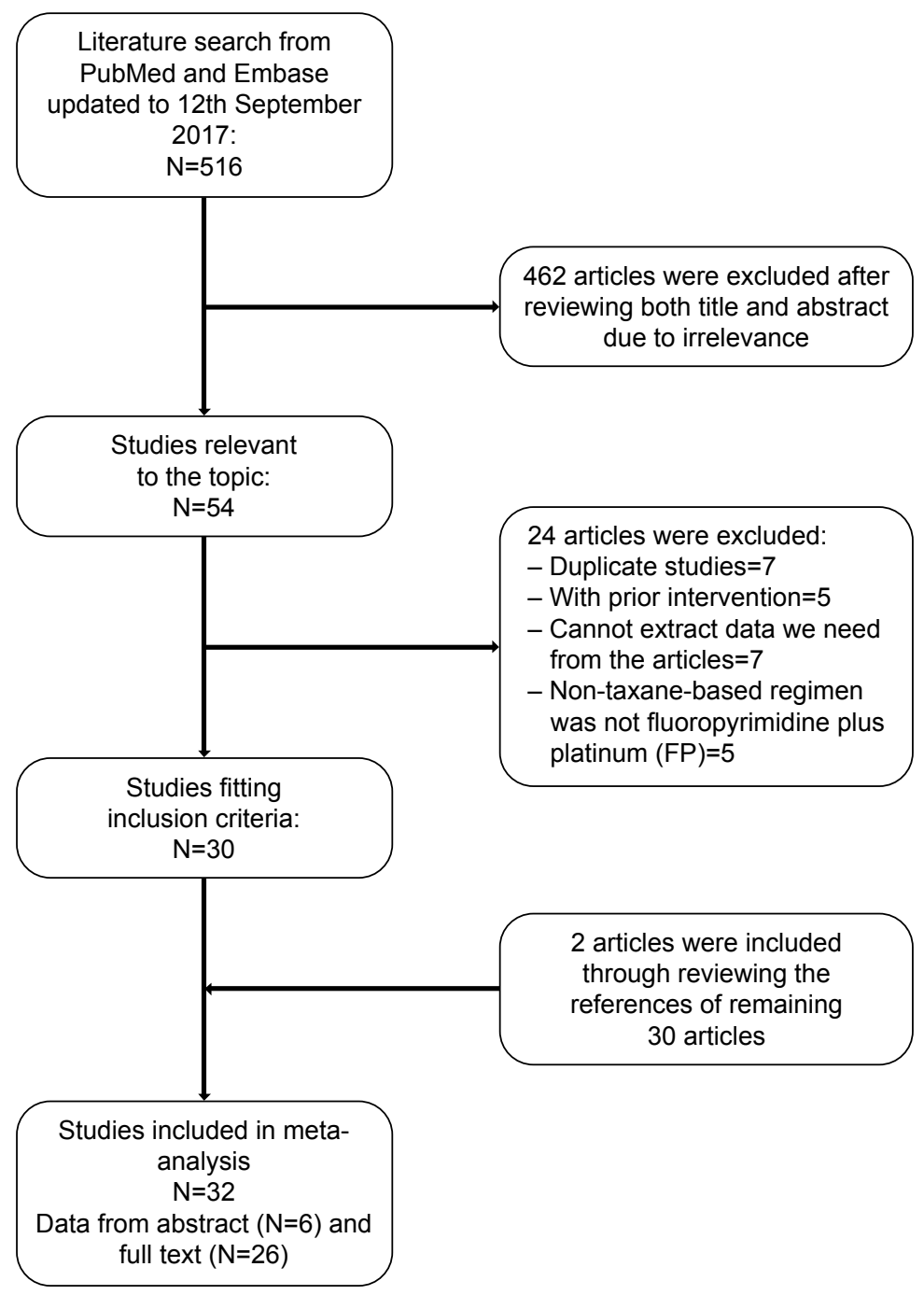

Figure I The flow chart of study selection. 


\begin{tabular}{l|l|l|l|l|l|l|l}
\hline $\begin{array}{l}\text { Chemotherapy } \\
\text { regimens }\end{array}$ & $\begin{array}{l}\text { Median radiation } \\
\text { dose (taxane/non- } \\
\text { taxane, Gy) }\end{array}$ & $\begin{array}{l}\text { Treatment } \\
\text { strategy }\end{array}$ & $\begin{array}{l}\text { Pathological } \\
\text { types }\end{array}$ & $\begin{array}{l}\text { Clinical } \\
\text { stage }\end{array}$ & $\begin{array}{l}\text { Research } \\
\text { type }\end{array}$ & Quality & Reference \\
\hline $\begin{array}{l}\text { DTX+5-FU+DDP vs } \\
\text { 5-FU+DDP }\end{array}$ & - & NACT & SCC & $11-I V$ & CS & 7 & 15 \\
$\begin{array}{l}\text { PTX+CBP vs } \\
\text { 5-FU+DDP }\end{array}$ & - & NACT & - & - & CS & 6 & 22 \\
$\begin{array}{l}\text { PTX+DDP vs } \\
\text { S-I+DDP }\end{array}$ & 60 & CCRT & SCC & $11-I V a$ & CS & 7 & 24 \\
$\begin{array}{l}\text { DTX+5-FU+DDP vs } \\
\text { 5-FU+DDP } \\
\text { PTX+5-FU vs }\end{array}$ & - & NACT & SCC & $11-I V$ & CS & 6 & $4 I$ \\
5-FU+DDP \\
$\begin{array}{l}\text { PTX+CBP vs } \\
\text { 5-FU+DDP }\end{array}$
\end{tabular}

(CR: pooled $\mathrm{OR}=0.52,95 \% \mathrm{CI}=0.21-1.28, P=0.15$; ORR: pooled $\mathrm{OR}=0.26,95 \% \mathrm{CI}=0.14-0.49, P<0.0001$; DCR: pooled $\mathrm{OR}=0.37,95 \% \mathrm{CI}=0.16-0.84, P=0.02$; Figure $2 \mathrm{~A}-\mathrm{C}$ ). Based on these results, we investigated whether the better clinical response in taxane-based NACT translated into higher $\mathrm{R} 0$ resection and $\mathrm{pCR}$ rates, and long-term survival. Our analysis showed that no difference was found in $\mathrm{R} 0$ resection (pooled $\mathrm{OR}=1.31,95 \% \mathrm{CI}=0.50-3.42, P=0.58$ ) between taxane-based NACT and FP NACT (Figure 2D). However, taxane-based NACT had higher pCR rates (pooled $\mathrm{HR}=0.45,95 \% \mathrm{CI}=0.21-0.94, P=0.03$; Figure $2 \mathrm{E})$ and better outcomes in PFS (pooled HR $=0.58,95 \% \mathrm{CI}=0.43-0.80$, $P=0.0008$; Figure 2F) and $\mathrm{OS}$ (pooled $\mathrm{HR}=0.50,95 \%$ $\mathrm{CI}=0.37-0.68, P<0.0001$; Figure $2 \mathrm{G}$ ) when compared to FP NACT. Based on these seven studies, we could find that taxane-based NACT caused more grade 3/4 neutropenia (pooled $\mathrm{OR}=13.28,95 \% \mathrm{CI}=1.37-129.01, P=0.03$ ) and diarrhea (pooled $\mathrm{OR}=5.50,95 \% \mathrm{CI}=1.88-16.05, P=0.002$ ) when compared with FP NACT (Figure S3). The prevalence of grade 3/4 anemia, leukopenia, thrombocytopenia, anorexia, nausea, and vomiting was similar (Table S3).

\section{Taxane-based NACRT therapy and FP NACRT therapy showed similar efficacy and toxicity in EC patients}

Including three studies that enrolled patients who received either taxane-based NACRT or taxane-based dCRT, a total of 13 studies were analyzed, among which 10 were comparisons of taxane plus platinum (TP) and FP, two compared TPF and FP, and one was a comparison of docetaxel and FP. There were no significant differences in ORR (pooled $\mathrm{OR}=1.06$, 95\% CI $=0.63-1.77, P=0.83$ ), DCR (pooled $\mathrm{OR}=1.04,95 \%$ $\mathrm{CI}=0.46-2.33, P=0.93$ ), R0 resection (pooled $\mathrm{OR}=0.76$, 95\% CI $=0.30-1.96, P=0.58$ ), $\mathrm{pCR}$ (pooled $\mathrm{OR}=1.15$, 95\% CI $=0.90-1.49, P=0.27$ ), PFS (pooled $\mathrm{HR}=1.25,95 \%$ $\mathrm{CI}=0.42-3.72, P=0.69)$ and $\mathrm{OS}$ (pooled $\mathrm{HR}=0.91,95 \%$ $\mathrm{CI}=0.69-1.20, P=0.52)$ between the taxane-based NACRT group and FP NACRT group (Table S2).

Between taxane-based NACRT and FP NACRT, there were no significant differences in grade $3 / 4$ anemia (pooled $\mathrm{OR}=0.95,95 \% \mathrm{CI}=0.33-2.71, P=0.92$ ), leukopenia (pooled $\mathrm{OR}=1.67,95 \% \mathrm{CI}=0.34-8.18 P=0.53$ ), neutropenia (pooled $\mathrm{OR}=2.18,95 \% \mathrm{CI}=0.42-11.29$, $P=0.35$ ), thrombocytopenia (pooled $\mathrm{OR}=0.21,95 \%$ $\mathrm{CI}=0.01-2.88, P=0.24$ ), nausea (pooled $\mathrm{OR}=0.61,95 \%$ $\mathrm{CI}=0.18-2.13, P=0.44$ ), vomiting (pooled $\mathrm{OR}=0.49$, 95\% CI $=0.23-1.05, P=0.07$ ), diarrhea (pooled $\mathrm{OR}=0.26,95 \% \mathrm{CI}=0.01-6.51, P=0.41$ ), esophagitis (pooled $\mathrm{OR}=1.43,95 \% \mathrm{CI}=0.81-2.51, P=0.21)$ or pneumonia (pooled OR=0.38, 95\% CI=0.08-1.93, $P=0.24$ ) (Table $\mathrm{S} 3$ ).

\section{Taxane-based dCRT results in better disease control and long-term survival and also causes severe toxicity more frequently compared with FP dCRT}

There were 14 studies (TP vs FP: 13 studies; paclitaxel+5FU vs FP: one study) altogether comparing taxane-based regimens with FP regimen in patients who received dCRT. Additionally, the study by Katada et al ${ }^{17}$ related to NACT 
also reported some data on clinical responses in some patients who just received dCRT. Data for short-term clinical responses (CR, ORR, and DCR) were extracted and it showed that taxane-based regimens were better than the FP regimen $(\mathrm{CR}$ : pooled $\mathrm{OR}=0.61,95 \% \mathrm{CI}=0.42-0.88, P=0.009$;
ORR: pooled $\mathrm{OR}=0.60,95 \% \mathrm{CI}=0.44-0.81, P=0.001$; and DCR: pooled $\mathrm{OR}=0.49,95 \% \mathrm{CI}=0.29-0.82, P=0.007$; Figure $3 \mathrm{~A}-\mathrm{C})$. Moreover, patients who received taxane-based dCRT had significantly better PFS (pooled $\mathrm{HR}=0.76,95 \%$ $\mathrm{CI}=0.67-0.88, P=0.0001$, Figure $3 \mathrm{D})$. However, we failed to

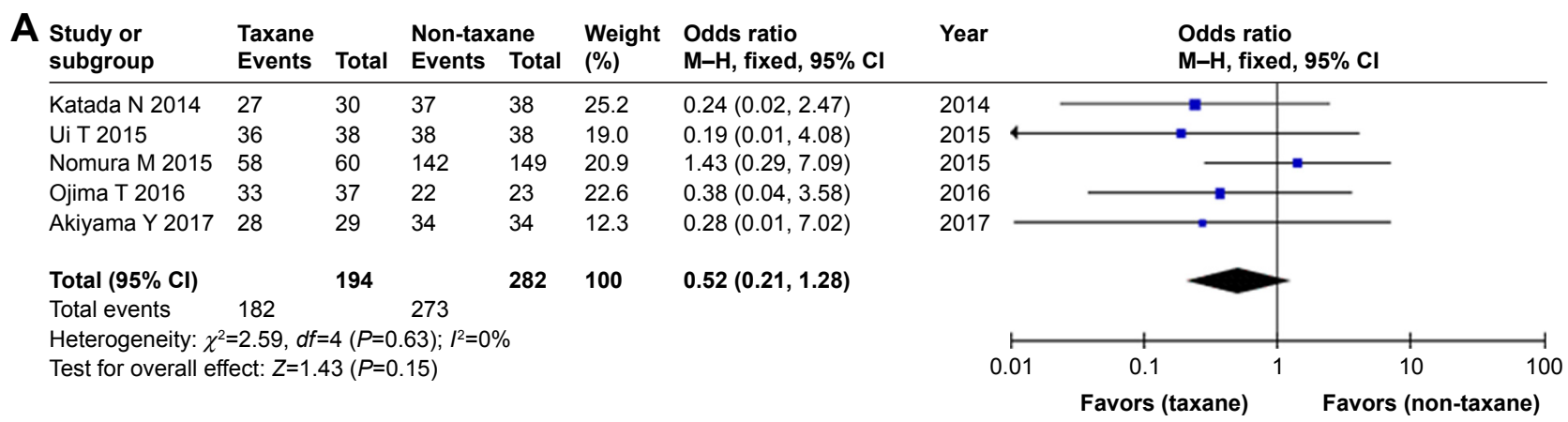

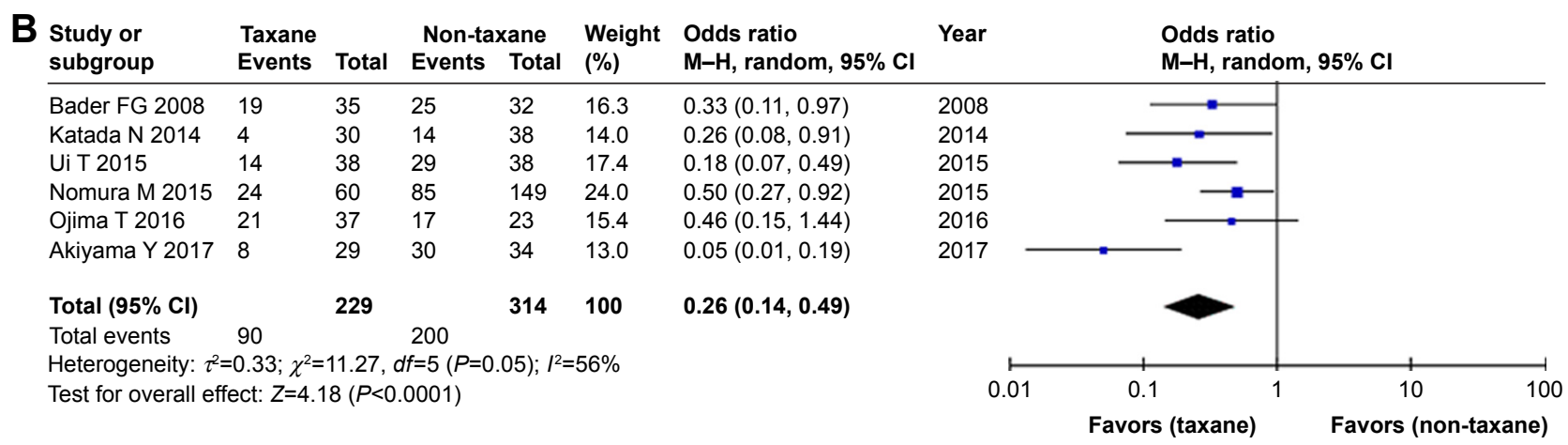

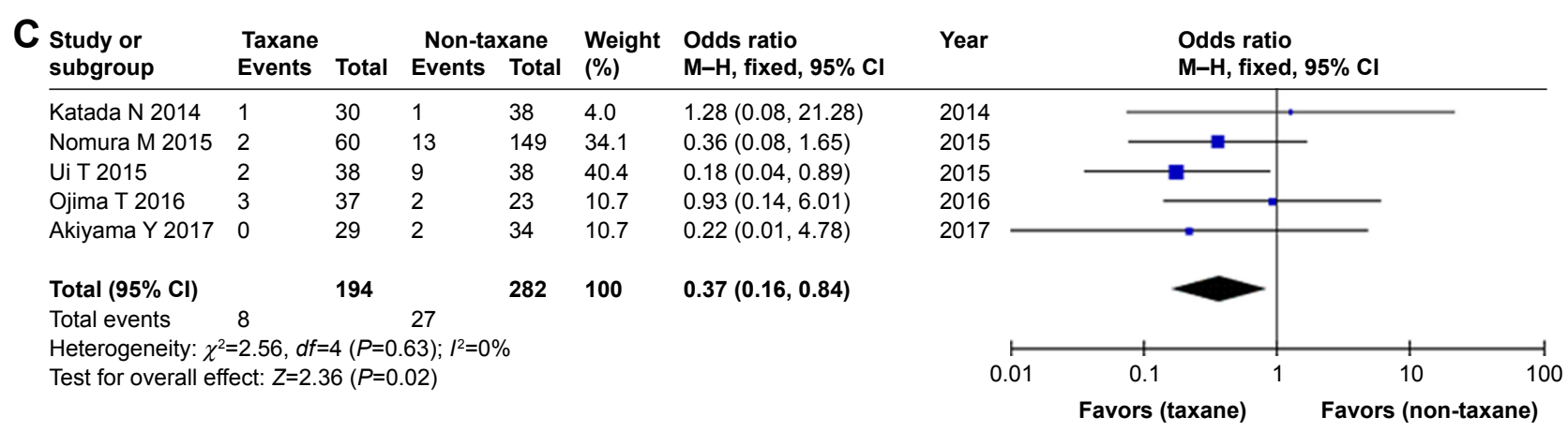

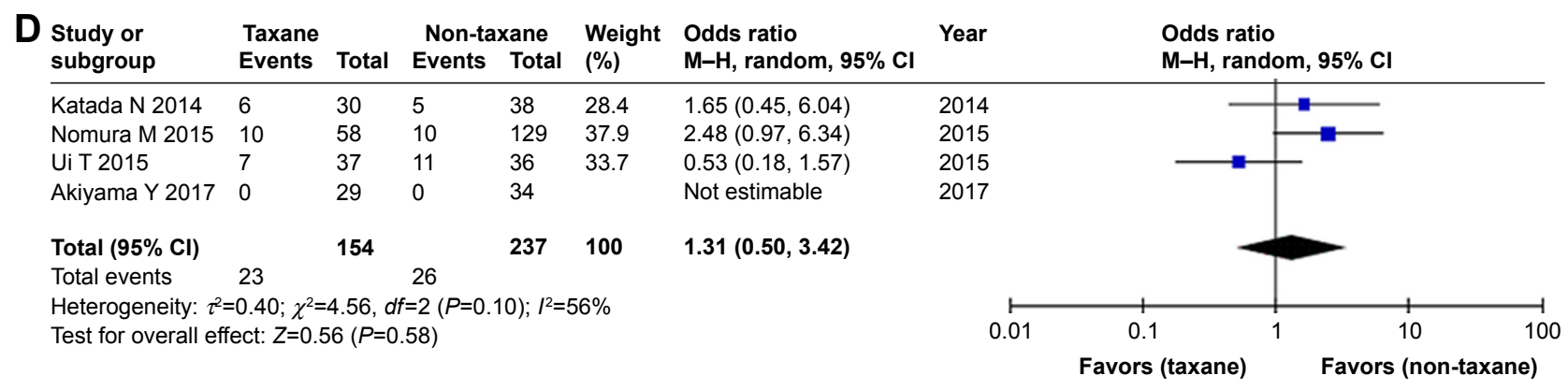

Figure 2 (Continued) 


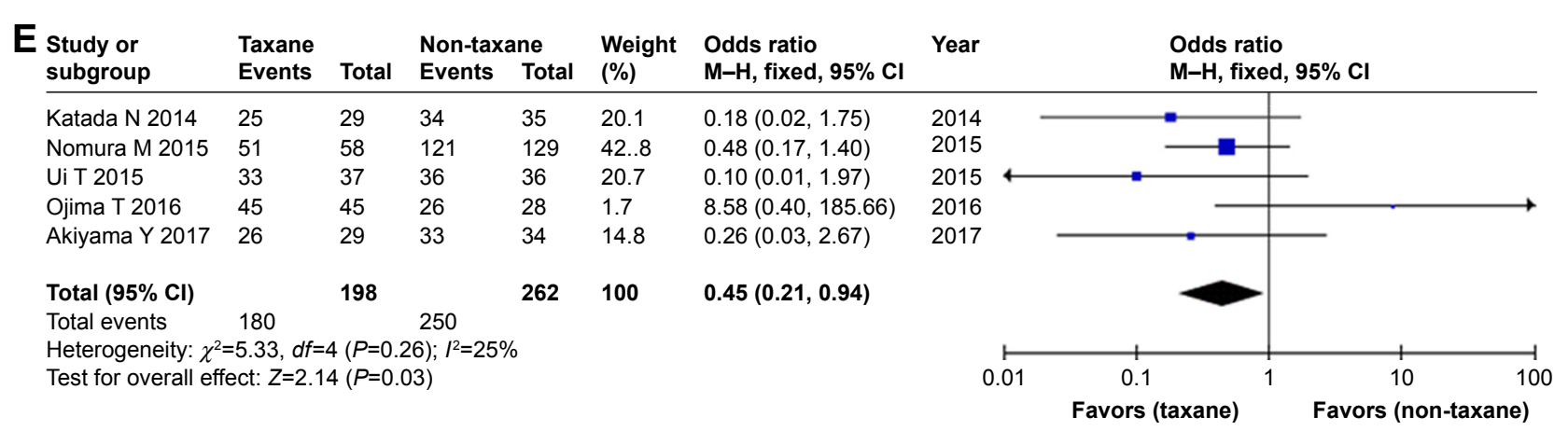

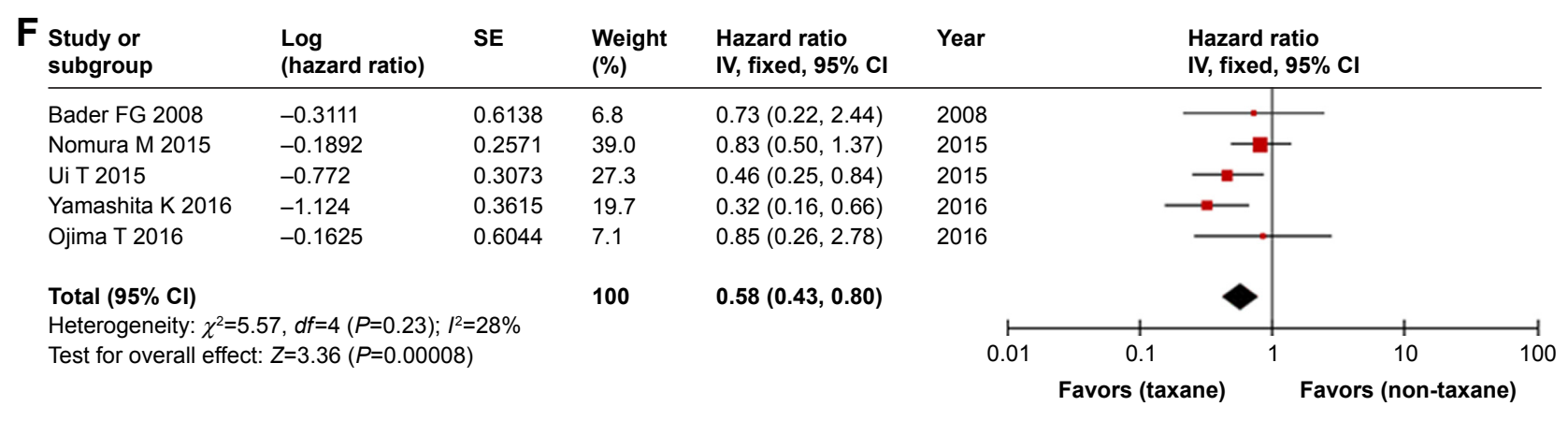

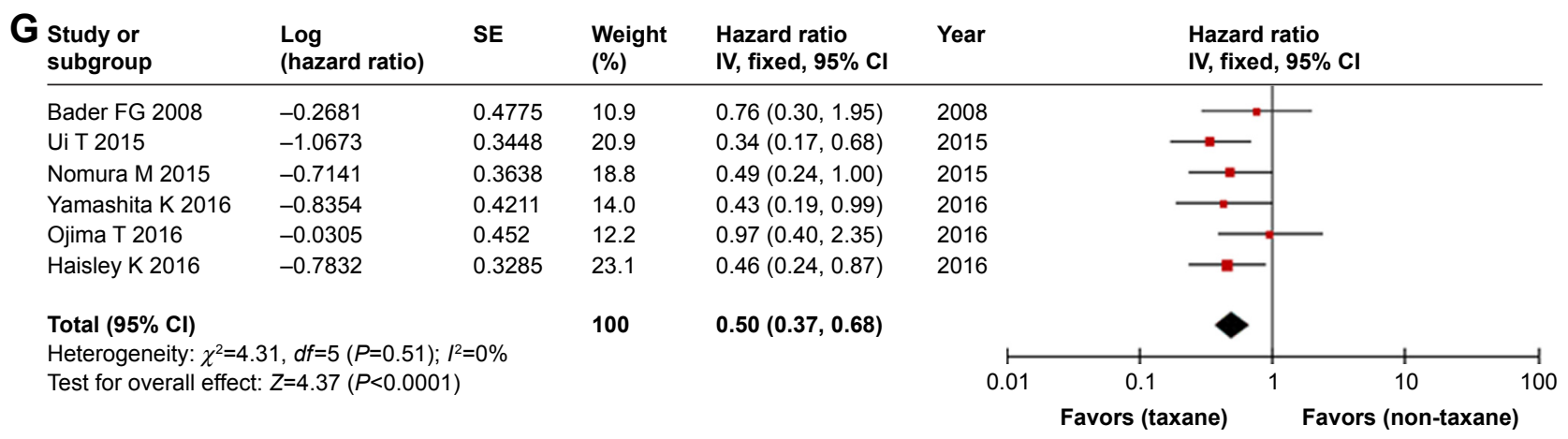

Figure 2 Analyses of curative effects between taxane-based NACT and FP based NACT in EC. (A) CR; (B) ORR; (C) DCR; (D) R0 resection; (E) PCR; (F) PFS; and (G) OS Abbreviations: CR, complete response; DCR, disease control rate; EC, esophageal cancer; NACT, neoadjuvant chemotherapy; ORR, objective response rate; OS, overall survival; PCR, pathological complete response; PFS, progression free survival; FP, fluoropyrimidine plus platinum.

find any benefit on $\mathrm{OS}$ (pooled $\mathrm{HR}=0.91,95 \% \mathrm{CI}=0.70-1.17$, $P=0.44$, Figure $3 \mathrm{E}$ ) in taxane-based dCRT.

Patients receiving taxane-based dCRT tend to have higher incidence rates of grade $3 / 4$ leukopenia (pooled $\mathrm{OR}=1.85$, 95\% $\mathrm{CI}=1.34-2.55, P=0.0002$ ) and pneumonia (pooled $\mathrm{OR}=2.32,95 \% \mathrm{CI}=1.23-4.38, P=0.009)$ when compared to FP dCRT. There was less grade $3 / 4$ nausea $(\mathrm{OR}=0.03,95 \%$ $\mathrm{CI}=0.00-0.21, P=0.0005)$ and vomiting $(\mathrm{OR}=0.04,95 \%$ $\mathrm{CI}=0.01-0.19, P<0.0001)$ in taxane-based therapy. However, it must be noted that this was based on only one study that compared paclitaxel plus $5-\mathrm{FU}$ with $\mathrm{CF},{ }^{44}$ and that cisplatin frequently causes gastrointestinal toxicity (Figure S4).

\section{Clinical benefits of taxane-based therapy in Esophageal squamous cell carcinoma (ESCC) patients}

ESCC is the most common type of EC in Asia and Eastern Europe. ${ }^{1}$ In ESCC, taxanes were shown to be highly effective in several clinical trials. ${ }^{45-48}$ Thus, we performed a separate analysis on the clinical benefits of taxane-based regimens in ESCC.

In ESCC patients who received taxane-based therapy NACT, there were four studies (TPF vs FP) comparing curative effects of two different regimens. We found that taxane-based NACT produced better ORR (pooled $\mathrm{OR}=0.26$, $95 \% \mathrm{CI}=0.10-0.67, P=0.005$ ) and $\mathrm{OS}$ (pooled $\mathrm{HR}=0.57$, 


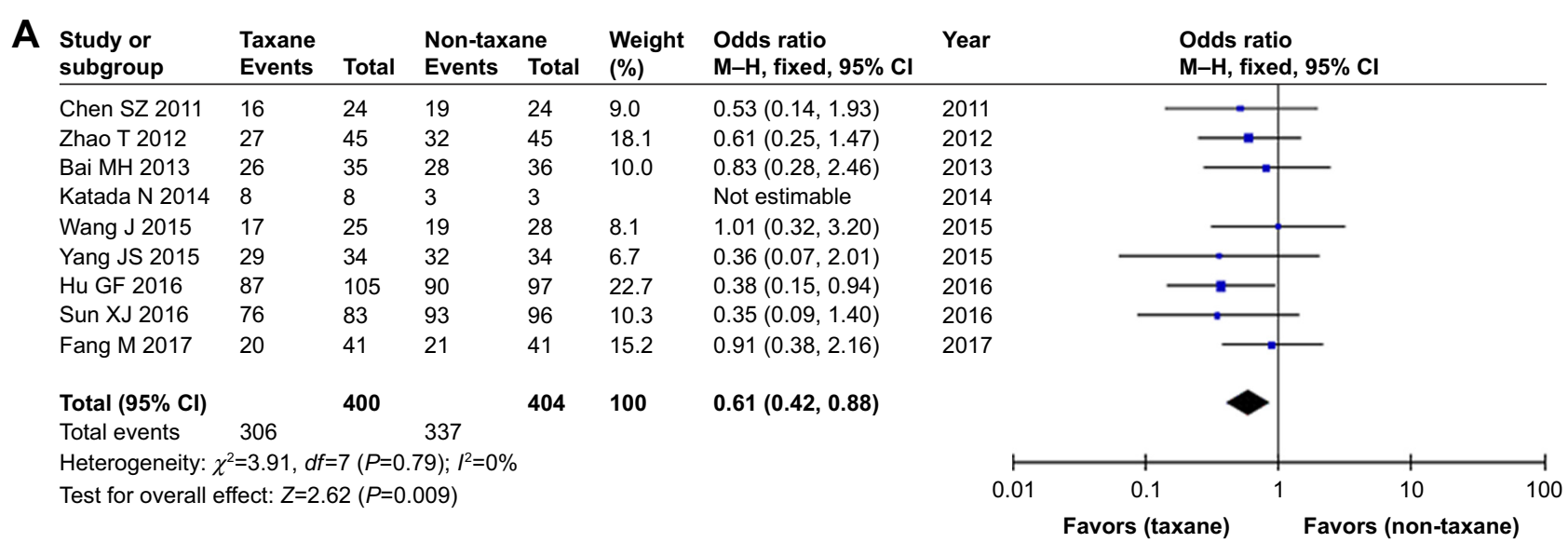

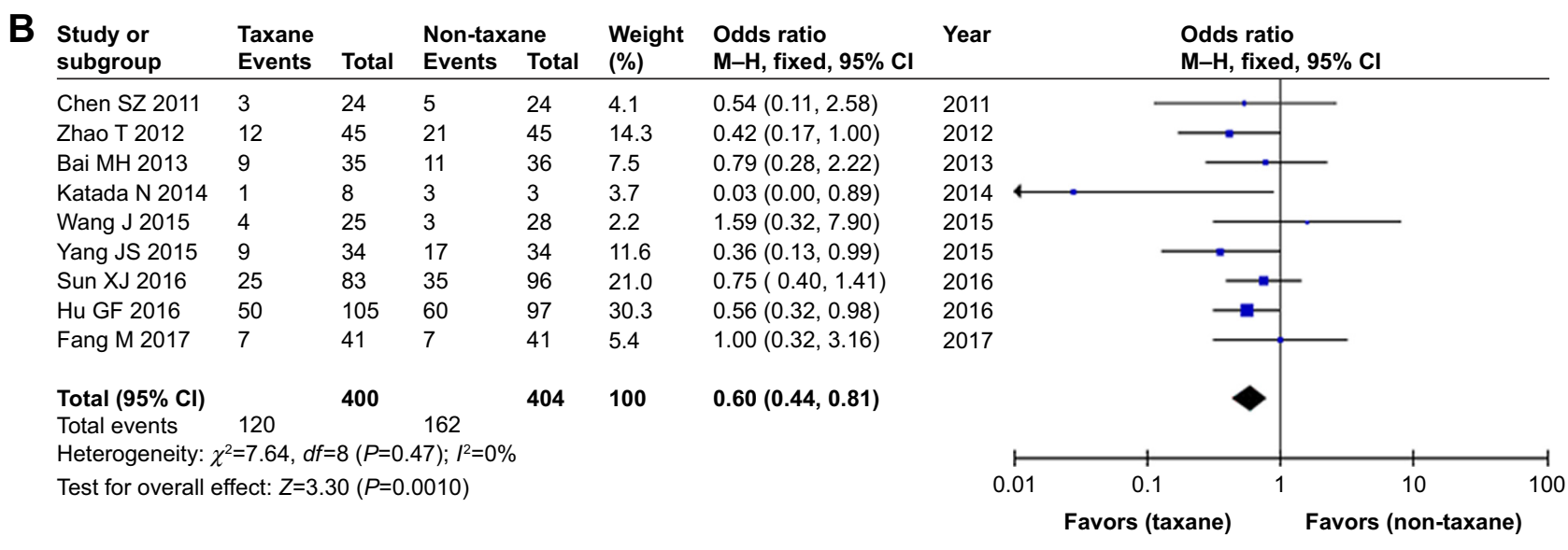

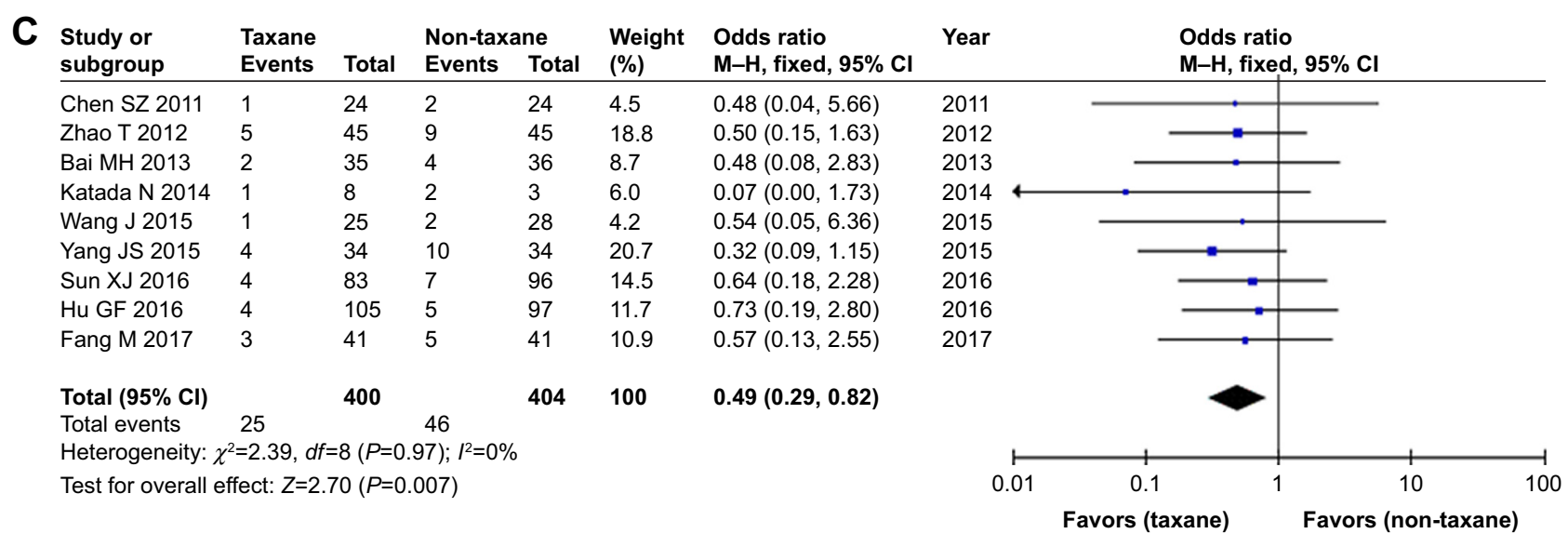

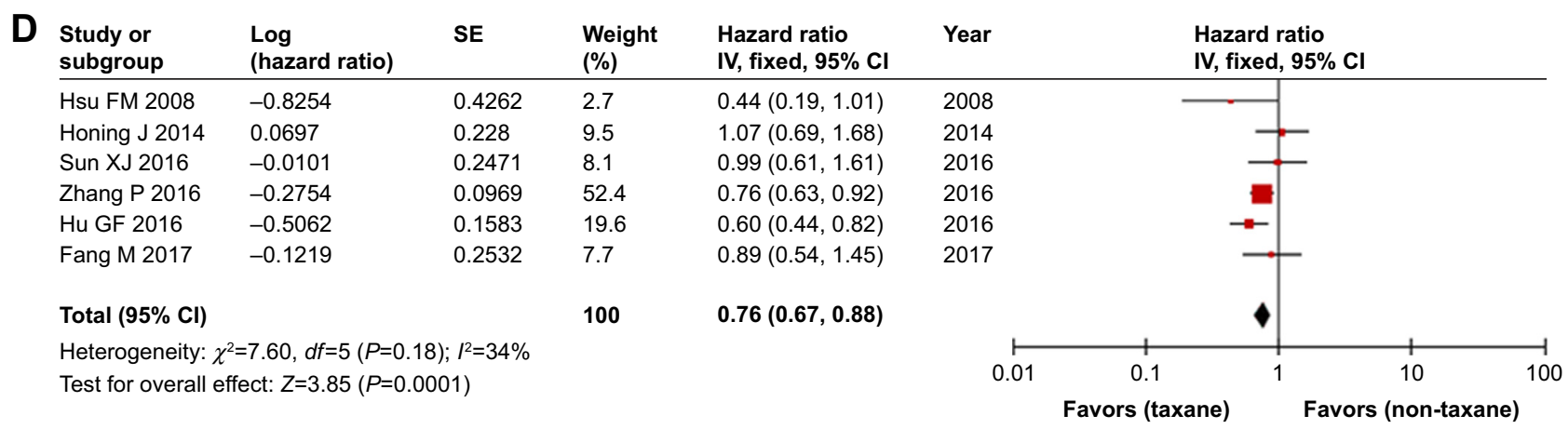

Figure 3 (Continued) 


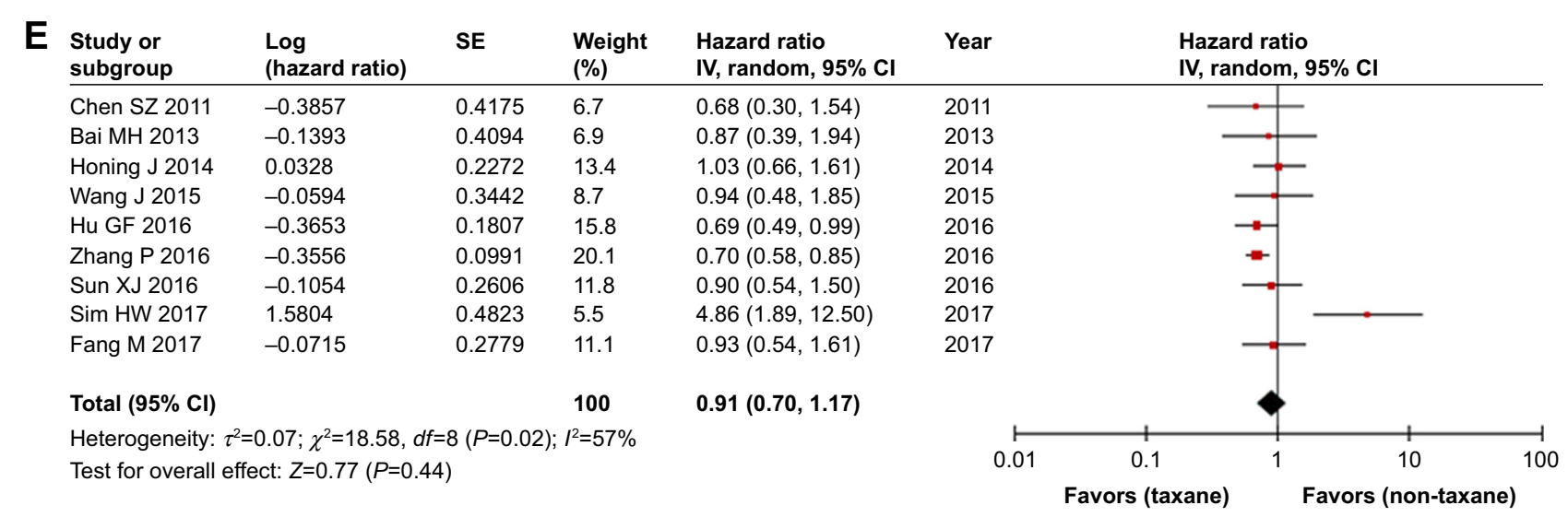

Figure 3 Analyses of curative effects between taxane-based dCRT and FP-based dCRT in EC. (A) CR; (B) ORR; (C) DCR; (D) PFS; and (E) OS.

Abbreviations: CR, complete response; DCR, disease control rate; dCRT, definitive chemoradiotherapy; EC, esophageal cancer; ORR, objective response rate; OS, overall survival; PFS, progression free survival.

$95 \% \mathrm{CI}=0.36-0.90, P=0.02)$. There was a higher frequency of grade $3 / 4$ leukopenia $(\mathrm{OR}=35.00,95 \% \mathrm{CI}=2.01-610.34$, $P=0.01$ ), neutropenia (pooled $\mathrm{OR}=33.71,95 \% \mathrm{CI}=9.58$ 118.7, $P=0.002$ ), and diarrhea (pooled $\mathrm{OR}=10.76,95 \%$ $\mathrm{CI}=1.86-62.07, P=0.008)$ (Tables S2 and $\underline{\mathrm{S} 3}$ ).

In ESCC patients who received taxane-based therapy NACRT, we found that taxane-based NACRT could bring about better pCR (pooled $\mathrm{OR}=0.50,95 \% \mathrm{CI}=0.28-0.89$, $P=0.02)$ and $\mathrm{OS}(\mathrm{HR}=0.51,95 \% \mathrm{CI}=0.28-0.93, P=0.03)$. Grade 3/4 leukopenia $(\mathrm{OR}=4.79,95 \% \mathrm{CI}=1.52-15.08$, $P=0.007)$ was more frequent when compared with FP NACRT (Tables S2 and $\underline{\mathrm{S}}$ ). No significant differences were found in CR, ORR, DCR, R0 resection, PFS, or other adverse events (Tables $\mathrm{S} 2$ and $\underline{\mathrm{S} 3}$ ).

In ESCC patients who received taxane-based therapy dCRT, we analyzed nine studies comparing TP with FP and one study comparing paclitaxel+5-FU with FP. Taxane-based dCRT resulted in better short-term clinical response (CR: pooled $\mathrm{OR}=0.57,95 \% \mathrm{CI}=0.38-0.85, P=0.006$; ORR: pooled $\mathrm{OR}=0.58,95 \% \mathrm{CI}=0.42-0.79, P=0.0005$; and DCR: pooled $\mathrm{OR}=0.49,95 \% \mathrm{CI}=0.29-0.893, P=0.008)$ and survival (PFS: pooled $\mathrm{HR}=0.74,95 \% \mathrm{CI}=0.64-0.85, P<0.0001$; and $\mathrm{OS}$ : pooled $\mathrm{HR}=0.73,95 \% \mathrm{CI}=0.63-0.85, P<0.0001)$ compared to FP dCRT (Table S2). However, taxane-based dCRT caused higher rates of grade $3 / 4$ leukopenia (pooled $\mathrm{OR}=1.80,95 \%$ $\mathrm{CI}=1.30-2.49, P=0.0004$ ) and pneumonia (pooled $\mathrm{OR}=2.32$, 95\% CI=1.23-4.38, $P=0.009$; Table S3).

\section{Discussion}

Nowadays, meta-analysis has become a very popular and powerful tool in evaluating the benefit or disadvantage of an intervention. Through pooling data from quantities of individual studies, meta-analysis could overcome limitations of small sample sizes or rare outcomes and increase the generalizability of study results. In addition, meta-analysis uses a process of computing weighted averages, and more accurate results will be assigned more weight in the computation of average, ${ }^{49}$ which, to some extent, eliminates the influence of different populations of patients and other biases which come with cohort studies. Therefore, it is more precise in estimating effects of interest when compared to an individual study. ${ }^{49}$ In our present study, we use the meta-analysis to investigate the benefits of taxane-based first-line therapy in the treatment of EC.

Taxanes, including paclitaxel and docetaxel, have been approved by the US Food and Drug Administration (FDA) for the treatment of many malignancies, including ovarian cancer, esophageal cancer, breast cancer, non-small-cell lung cancer and other types of malignancies. ${ }^{50}$ This class of drugs promotes the formation of stable microtubules, prolongs the G2 and M phases of the cell cycle, induces cell apoptosis, and inhibits the motility of cancer cells. ${ }^{51}$ In the treatment of $\mathrm{EC}$, the FP regimen has been considered standard and has been widely used for more than 30 years. Recently, taxanes combined with platinum also produced good responses and was another first-line chemotherapeutic regimen. To date, there is no consensus as to whether taxane-based chemotherapy is better than FP.

Our results show that patients who received NACT and dCRT benefit more from taxane-based therapy than from FP treatment. In patients who received NACRT therapy, taxanebased treatment and FP therapy showed similar efficacy and toxicities. With the SCC subtype, taxane-based therapy had a higher activity than FP therapy in NACT, dCRT, and NACRT. However, taxane-based regimens were associated with higher rates of severe leukopenia, neutropenia, diarrhea, 
or pneumonia when compared with the FP regimen. The cumulative incidence rates of grade 3/4 leukopenia, neutropenia, diarrhea, and pneumonia were $28 \%, 50 \%, 5 \%$, and $3 \%$, respectively, in the taxane group by using $\mathrm{R}$ software (Figure S5). Fortunately, there was no significant difference in treatment-related deaths between the taxane group and FP group (pooled $\mathrm{OR}=0.73,95 \% \mathrm{CI}=0.38-1.40, P=0.34$, Figure S6).

The primary treatment options for EC include preoperative chemoradiotherapy/chemotherapy, esophagectomy, and definitive CCRT. Among these options, neoadjuvant therapy, including chemotherapy and chemoradiotherapy followed by surgery, has been proven to improve OS in patients with resectable EC when compared to surgery alone. ${ }^{5}$ In the Medical Research Council OEO2 trial, patients receiving NACT with CF had longer DFS and OS than those receiving surgery alone..$^{52}$ Presently, only CF is recommended for preoperative chemotherapy for EC, and it is only used for adenocarcinoma of the thoracic esophagus or esophagogastric junction cancers (EGJ) and not in SCC. However, in the OGSG1003 trial, patients with ESCC receiving taxane-based NACT had a 2-year RFS of $64.1 \%$ and a 2 -year OS of $78.6 \% .{ }^{53}$ Our results showed that taxane-based NACT is associated with better short-term tumor response and long-term survival benefits compared with FP NACT. Similar results were also found in ESCC patients. In this case, taxane-based regimens could be useful for preoperative chemotherapy in EC. However, more RCTs are needed to provide enough evidence.

NACRT is another important option for EC patients. The preferred regimens classified as category one for preoperative chemoradiation in EC include paclitaxel plus carboplatin and fluorouracil plus oxaliplatin (NCCN Guidelines, Version 2, 2018). Data from individual studies showed that both the median PFS and OS in patients who received NACRT with paclitaxel and carboplatin from the CROSS trial were longer than those in patients who received NACRT with fluorouracil and cisplatin from the FFCD 9901 trial (PFS: 37.7 months vs 27.8 months; OS: 48.6 months vs 31.8 months). ${ }^{4,54}$ However, clinical stage and tumor location of patients included in the CROSS trial are different from those of the FFCD9901. In the CROSS trial, there were $22 \%$ patients located in esophagogastric junction, $84 \%$ patients with cT3 and $65 \%$ patients with $\mathrm{cN} 1$. In contrast, there were $100 \%$ patients with esophageal cancer, $15.3 \%$ patients with cT3, and $29.6 \%$ patients with cN1 in FFCD9901. The median age (60 years in CROSS vs 58.1 years in FFCD9901), radiation dose (41.4 Gy in CROSS vs 45 Gy in FFCD9901), and WHO performance score were similar. It was more interesting that patients with more advanced stage in the CROSS study had longer PFS and OS than patients with a relatively earlier stage in the FFCD 9901 trial. Although this result was just based on the very simple comparison of reported data, we could find the difference of chemotherapeutic regimes between two studies. As a result, it is worthy of analyzing whether different chemotherapeutic regimes affect the survival. In our study, no significant differences were found between taxane-based regimens and FP regimen in the subgroup of NACRT. Between NACT and NACRT, the main difference was whether radiation intervention was used. Therefore, it seems that radiation is the main confounding factor and it is necessary to analyze whether additional intervention of radiation could cause severe adverse events which may hide the benefit of taxane-based chemotherapy. We found that no significant differences were found in grade 3/4 leukopenia and neutropenia, two common toxicities in our study (Figure S7). So, at least for now, we couldn't attribute the absence of taxane-based chemotherapeutic benefit to the advent of severe adverse events caused by NACRT. We hope the PROTECT-1402 study will give us some answers about this issue. Furthermore, whether the benefit of taxanebased chemotherapy can be hidden by radiation still needs further discussion.

While we did not find any benefits of taxane-based NACRT in all EC patients, we did see that taxane-based NACRT improved pCR and OS in SCC when compared with FP regimens. Similar to our results, Huang et $\mathrm{al}^{55}$ found that the HRs $(95 \% \mathrm{CI})$ of paclitaxel plus platinum regimen in the entire, SCC, and adenocarcinoma population were 0.80 (0.60-1.06), 0.61 (0.41-0.91), and 0.91 (0.61-1.36), respectively, when compared with FP NACRT. Furthermore, our results also support the rationale behind the ongoing randomized phase 2 PROTECT-1402 trial (NCT02359968), which tries to compare preoperative chemoradiation with either paclitaxel plus carboplatin or FOLFOX in esophageal and junctional cancer with either adenocarcinoma or squamous cell carcinoma.

For unresectable EC, definitive CCRT has become a standard treatment since the results of RTOG 85-01 were reported. ${ }^{6}$ Several studies conducted thereafter confirmed the efficacy of dCRT in the treatment of EC. FP was used as a standard concurrent chemotherapeutic regimen in most studies and classified as category one level recommendation. In recent years, taxane plus carboplatin/cisplatin was also recommended based on a series of clinical trials, ${ }^{56,57}$ but this combination is the only category $2 \mathrm{~A}$ recommendation. Although both regimens are deemed effective, there is 
currently no consensus as to whether taxane-based dCRT regimens are better than FP dCRT. The results of several studies are conflicting. ${ }^{11,17,21,24}$ Schellenberg et $\mathrm{al}^{42}$ reported that cisplatin+5 FU or carboplatin+paclitaxel concurrent with radiation showed no difference in PFS, while Hu et $\mathrm{al}^{13}$ found that both median PFS and OS in the taxane group were significantly better than in the FP group (median PFS, 15.9 vs 13.0 months and median OS, 33.9 vs 23.1 months, respectively). In our study, we found that taxane-based therapy produced better clinical response and PFS, but failed to show an improvement in OS. As for adverse events, taxane-based dCRT showed a higher incidence of grade 3/4 leukopenia and pneumonia.

While the most common histologic type of EC in patients from the Western countries is adenocarcinoma, most Asian patients would have SCC. ${ }^{58}$ In a multicenter Phase II trial of sequential preoperative induction chemotherapy and chemoradiation, ${ }^{59}$ it was reported that patients with SCC had better response rates and survival compared to patients with adenocarcinoma. This, however, was not statistically significant. In patients with SCC, however, our results similarly showed that taxane-based dCRT resulted not only in better clinical responses, but also in longer PFS and OS. With these results, it might be worth considering the re-evaluation of the recommendations on the use of taxanes in dCRT.

It must be noted, however, that, while 32 articles were included in our meta-analysis, data could be extracted only from what was made available in the abstract and the published paper. Some data for OS and PFS were extracted from Kaplan-Meier curves and calculated indirectly to get HRs. Significant heterogeneities were found in many subgroups, and many subgroup analyses took into account $<10$ trials. Furthermore, among all the studies included, there were only a few RCTs, and some of the studies were only of medium quality. The incomplete data might have restricted our analysis on the benefits of taxane-based regimens in patients with adenocarcinoma.

\section{Conclusion}

Taxane-based regimens could produce better clinical response and outcomes, but are associated with increased toxicity (mainly leukopenia, neutropenia, and diarrhea) compared to FP regimens. EC patients who received NACT, dCRT, or those with an SCC benefit more from taxane-based therapy. In the future, more trials should be conducted, especially in SCC, to define the best niche for taxane-based regimens in the treatment of EC.

\section{Acknowledgments}

This work is supported by the project of Jiangsu Provincial Medical Youth Talent (QNRC2016319), National Natural Science Foundation of China (81302015), Social development project of Yangzhou City (YZ2014181), and the Natural Science Foundation of Yangzhou City (YZ2014044).

\section{Disclosure}

The authors report no conflicts of interest in this work.

\section{References}

1. Torre LA, Bray F, Siegel RL, Ferlay J, Lortet-Tieulent J, Jemal A Global Cancer statistics, 2012. CA Cancer J Clin. 2015;65(2):87-108.

2. Global Burden of Disease Cancer Collaboration, Fitzmaurice C, Allen C, et al. Global, regional, and National cancer incidence, mortality, years of life lost, years lived with disability, and Disability-Adjusted life-years for 32 cancer groups, 1990 to 2015: a systematic analysis for the global burden of Disease Study. JAMA Oncol. 2017;3(4):524-548.

3. Chen W, Zheng R, Baade PD, et al. Cancer statistics in China, 2015. CA Cancer J Clin. 2016;66(2):115-132.

4. Shapiro J, van Lanschot JJB, Hulshof M, et al. Neoadjuvant chemoradiotherapy plus surgery versus surgery alone for oesophageal or junctional cancer (cross): long-term results of a randomised controlled trial. Lancet Oncol. 2015;16(9):1090-1098.

5. Sjoquist KM, Burmeister BH, Smithers BM, et al. Survival after neoadjuvant chemotherapy or chemoradiotherapy for resectable oesophageal carcinoma: an updated meta-analysis. Lancet Oncol. 2011;12(7):681-692.

6. Cooper JS, Guo MD, Herskovic A, et al. Chemoradiotherapy of locally advanced esophageal cancer: long-term follow-up of a prospective randomized trial (RTOG 85-01). Radiation therapy Oncology Group. JAMA. 1999;281(17):1623-1627.

7. Lagergren J, Lagergren P. Recent developments in esophageal adenocarcinoma. CA Cancer J Clin. 2013;63(4):232-248.

8. Siegel RL, Miller KD, Jemal A. Cancer statistics, 2018. CA Cancer J Clin. 2018;68(1):7-30.

9. Ter Veer E, Haj Mohammad N, van Valkenhoef G, et al. The efficacy and safety of first-line chemotherapy in advanced esophagogastric cancer: a network meta-analysis. J Natl Cancer Inst. 2016;108(10):djw166.

10. Ando N, Kato H, Igaki H, et al. A randomized trial comparing postoperative adjuvant chemotherapy with cisplatin and 5-fluorouracil versus preoperative chemotherapy for localized advanced squamous cell carcinoma of the thoracic esophagus (JCOG9907). Ann Surg Oncol. 2012;19(1):68-74.

11. Honing J, Smit JK, Muijs CT, et al. A comparison of carboplatin and paclitaxel with cisplatinum and 5-fluorouracil in definitive chemoradiation in esophageal cancer patients. Ann Oncol. 2014;25(3):638-643.

12. Sun X, Han S, Gu F, et al. A retrospective comparison of taxane and Fluorouracil-based chemoradiotherapy in patients with inoperable esophageal squamous cell carcinoma. J Cancer. 2016;7(9):1066-1073.

13. Hu G, Wang Z, Wang Y, et al. Comparison of cisplatinum/paclitaxel with cisplatinum/5-fluorouracil as first-line therapy for nonsurgical locally advanced esophageal squamous cell carcinoma patients. Drug Des Devel Ther. 2016;10:2129-2136.

14. Yamashita K, Katada N, Moriya H, et al. Neoadjuvant chemotherapy of triplet regimens of docetaxel/cisplatin/5-FU (dcf NAc) may improve patient prognosis of cStage II/III esophageal squamous cell carcinoma-propensity score analysis. Gen Thorac Cardiovasc Surg. 2016;64(4):209-215.

15. Ojima T, Nakamori M, Nakamura M, et al. Neoadjuvant chemotherapy with Divided-dose docetaxel, cisplatin and fluorouracil for patients with squamous cell carcinoma of the esophagus. Anticancer Res. 2016;36(2):829-834. 
16. Zhang P, Xi M, Li QQ, et al. Concurrent cisplatin and 5-fluorouracil versus concurrent cisplatin and docetaxel with radiotherapy for esophageal squamous cell carcinoma: a propensity score-matched analysis. Oncotarget. 2016;7(28):44686-44694.

17. Yang J-S, Wang T, Qiu M-Q, Li Q-L. Comparison of efficacy and toxicity profiles between paclitaxel/lobapoatin- and cisplatin/5-fluorouracil-based concurrent chemoradiotherapy of advanced inoperable oesophageal cancer. Intern Med J. 2015;45(7):757-761.

18. Nomura M, Oze I, Abe T, et al. Impact of docetaxel in addition to cisplatin and fluorouracil as neoadjuvant treatment for resectable stage III or T3 esophageal cancer: a propensity score-matched analysis. Cancer Chemother Pharmacol. 2015;76(2):357-363.

19. Ui T, Fujii H, Hosoya Y, et al. Comparison of preoperative chemotherapy using docetaxel, cisplatin and fluorouracil with cisplatin and fluorouracil in patients with advanced carcinoma of the thoracic esophagus. Dis Esophagus. 2015;28(2):180-187.

20. Zhao T, Chen H, Zhang T. Docetaxel and cisplatin concurrent with radiotherapy versus 5-fluorouracil and cisplatin concurrent with radiotherapy in treatment for locally advanced oesophageal squamous cell carcinoma: a randomized clinical study. Med Oncol. 2012;29(5):3017-3023.

21. Sim H-W, Chan BA, Natori A, et al. Comparison of chemoradiotherapy (crt) using carboplatin/paclitaxel (CP) versus cisplatin/5-FU (CF) for esophageal or gastroesophageal junctional (GEJ) cancer. J Clinl Oncol. 2017;35(15_suppl):4053.

22. Haisley K, Hart K, Nabavizadeh N. Neoadjuvant cisplatin/5-FU improves pathologic complete response and survival compared to carboplatin/paclitaxel in esophageal cancer. Dis Esophagus. 2016; 29:38A.

23. Thomay AA, Su S, Friedant AJ, et al. Freedom from recurrence after induction cisplatin/5-FU/RT versus carboplatin/paclitaxel/RT in patients with esophageal cancer. J Clin Oncol. 2014;32(3_suppl):126.

24. Fang M, Song T, Liang X, et al. Comparative study of cisplatin-based definitive concurrent chemoradiotherapy with S-1 versus paclitaxel for unresectable locally advanced esophageal squamous cell carcinoma. Oncotarget. 2017;8(23):37080-37090.

25. Zemanova M, Petruzelka L, Pazdro A, et al. Prospective non-randomized study of preoperative concurrent platinum plus 5-fluorouracil-based chemoradiotherapy with or without paclitaxel in esophageal cancer patients: long-term follow-up. Dis Esophagus. 2010;23(2):160-167.

26. Adelstein DJ, Rice TW, Rybicki LA, et al. Does paclitaxel improve the chemoradiotherapy of locoregionally advanced esophageal cancer? A nonrandomized comparison with fluorouracil-based therapy. J Clin Oncol. 2000;18(10):2032-2039.

27. Tierney JF, Stewart LA, Ghersi D, Burdett S, Sydes MR. Practical methods for incorporating summary time-to-event data into meta-analysis. Trials. 2007;8(1):16.

28. Higgins JP, Thompson SG, Deeks JJ, Altman DG. Measuring inconsistency in meta-analyses. BMJ. 2003;327(7414):557-560.

29. Blom RL, Sosef MN, Nap M, et al. Comparison of two neoadjuvant chemoradiotherapy regimens in patients with potentially curable esophageal carcinoma. Dis Esophagus. 2014;27(4):380-387.

30. Courrech Staal EF, Aleman BM, van Velthuysen ML, et al. Chemoradiation for esophageal cancer: institutional experience with three different regimens. Am J Clin Oncol. 2011;34(4):343-349.

31. Roof KS, Coen J, Lynch TJ, et al. Concurrent cisplatin, 5-FU, paclitaxel, and radiation therapy in patients with locally advanced esophageal cancer. Int J Radiat Oncol Biol Phys. 2006;65(4):1120-1128.

32. Hsu FM, Lin CC, Lee JM, et al. Improved local control by surgery and paclitaxel-based chemoradiation for esophageal squamous cell carcinoma: results of a retrospective non-randomized study. J Surg Oncol. 2008;98(1):34-41.

33. Bader FG, Lordick F, Fink U, et al. Paclitaxel in the neoadjuvant treatment for adeno carcinoma of the distal esophagus (AEG I). A comparison of two phase II trials with long-term follow-up. Onkologie. 2008;31(7):366-372.
34. Chen SZ, Chen XM, Ding Y, Wang XC, Zhang F, Mo KL. [Therapeutic effect of combined cisplatin and docetaxel vs fluorouracil regimen with concurrent radiotherapy on advanced esophageal carcinoma]. Nan Fang Yi Ke Da Xие Хие Bao. 2011;31(4):727-729. Chinese

35. Bai M, Wang B, Wang X, Ma H, Wang Y, Wang Z. Randomized trial of weekly docetaxel and cisplatin combined with concurrent 3DCRT in patients with locally advanced esophageal cancer. Chin Ger J Clin Oncol. 2013;12(8):361-364.

36. Kushida T, Nohara S, Yoshino K, et al. Utility of weekly docetaxel combined with preoperative radiotherapy for locally advanced esophageal cancer from pathological analysis. Dis Esophagus. 2014;27(4):368-373.

37. Katada N, Yamashita K, Katada C, et al. Neoadjuvant chemotherapy using concurrent Docetaxel/CDDP/5-FU (dcf) in esophageal squamous cell carcinoma and its short-term prognosis. Esophagus. 2014;11(3):173-181.

38. Wang J, Yu Y, Zhan B-H, Ge N, Zhou Q. Radiotherapy concurrent with paclitaxel plus cisplatin $v s$-fluorouracil plus cisplatin for esophageal cancer. World Chin J Digestol. 2015;23(24):3904.

39. Boggs DH, Tarabolous C, Morris CG, et al. Analysis of pathological complete response rates with paclitaxel-based regimens in trimodality therapy for esophageal cancer. Dis Esophagus. 2015;28(7):619-625.

40. Wu S, Chen MY, Luo JC, Wei L, Chen Z. [Comparison between docetaxel plus cisplatin and cisplatin plus fluorouracil in the neoadjuvant chemoradiotherapy for local advanced esophageal squamous cell carcinoma]. Zhonghua Zhong Liu Za Zhi. 2012;34(11):873-876. Chinese

41. Akiyama Y, Iwaya T, Endo F, et al. Investigation of operative outcomes of thoracoscopic esophagectomy after triplet chemotherapy with docetaxel, cisplatin, and 5-fluorouracil for advanced esophageal squamous cell carcinoma. Surg Endosc. 2018;32(1):391-399.

42. Schellenberg D, Peixoto R, Lee A, Lim HJ. A province-wide retrospective comparison of pathologic response and progression-free survival in locally advanced esophagus cancer patients treated with either cisplatin5FU (CF) or carboplatin-paclitaxel (CP) concurrent with radiation (XRT) with or without surgery. J Clin Oncol. 2014;32(3_suppl):107.

43. Berman AT, Barsky AR, Mick R, et al. Perioperative complications after neoadjuvant chemoradiation for locally advanced esophageal cancer: a comparison of platinum/5-FU (PF) and carboplatin/paclitaxel (Pt). Int J Radiat Oncol Biol Phys. 2014;90(1):S205.

44. Chen Y, Zhao K, Ye J, et al. Safety results of a phase 3 study of comparing paclitaxel plus 5-fluorouracil versus cisplatin plus 5-fluorouracil in chemoradiotherapy for locally advanced esophageal carcinoma (ESOShanghai 1). J Clin Oncol. 2017;35(15_suppl):4066.

45. Muro K, Hamaguchi T, Ohtsu A, et al. A phase II study of single-agent docetaxel in patients with metastatic esophageal cancer. Ann Oncol. 2004;15(6):955-959.

46. Albertsson M, Johansson B, Friesland S, et al. Phase II studies on docetaxel alone every third week, or Weekly in combination with gemcitabine in patients with primary locally advanced, metastatic, or recurrent esophageal cancer. Med Oncol. 2007;24(4):407-412.

47. Ajani JA, Ilson DH, Daugherty K, Pazdur R, Lynch PM, Kelsen DP. Activity of taxol in patients with squamous cell carcinoma and adenocarcinoma of the esophagus. J Natl Cancer Inst. 1994;86(14):1086-1091.

48. Ilson DH, Wadleigh RG, Leichman LP, Kelsen DP. Paclitaxel given by a Weekly 1-H infusion in advanced esophageal cancer. Ann Oncol. 2007;18(5):898-902.

49. Nordmann AJ, Kasenda B, Briel M. Meta-analyses: what they can and cannot do. Swiss Med Wkly. 2012;142:w13518.

50. Cragg GML, Kingston DGI, Newman DJ. Anticancer Agents from Natural Products. 2nd ed. Boca Raton: CRC Press; 2012.

51. Tishler RB, Schiff PB, Geard CR, Hall EJ. Taxol: a novel radiation sensitizer. Int J Radiat Oncol Biol Phys. 1992;22(3):613-617.

52. Allum WH, Stenning SP, Bancewicz J, Clark PI, Langley RE. Longterm results of a randomized trial of surgery with or without preoperative chemotherapy in esophageal cancer. J Clin Oncol. 2009;27(30): $5062-5067$. 
53. Yamasaki M, Yasuda T, Yano M, et al. Multicenter randomized phase II study of cisplatin and fluorouracil plus docetaxel (dcf) compared with cisplatin and fluorouracil plus adriamycin (ACF) as preoperative chemotherapy for resectable esophageal squamous cell carcinoma (OGSG1003). Ann Oncol. 2017;28(1):116-120.

54. Mariette C, Dahan L, Mornex F, et al. Surgery alone versus chemoradiotherapy followed by surgery for stage I and II esophageal cancer: final analysis of randomized controlled phase III trial FFCD 9901. $J$ Clin Oncol. 2014;32(23):2416-2422.

55. Huang TC, Hsu CH, Lin CC, Tu YK. Systematic review and network meta-analysis: neoadjuvant chemoradiotherapy for locoregional esophageal cancer. Jpn J Clin Oncol. 2015;45(11):1023-1028.

56. Day FL, Leong T, Ngan S, et al. Phase I trial of docetaxel, cisplatin and concurrent radical radiotherapy in locally advanced oesophageal cancer. Br J Cancer. 2011;104(2):265-271.
57. Li QQ, Liu MZ, Hu YH, Liu H, He ZY, Lin HX. Definitive concomitant chemoradiotherapy with docetaxel and cisplatin in squamous esophageal carcinoma. Dis Esophagus. 2010;23(3):253-259.

58. Zhang HZ, Jin GF, Shen HB. Epidemiologic differences in esophageal cancer between Asian and Western populations. Chin J Cancer. 2012; 31(6):281-286.

59. Ruhstaller T, Widmer L, Schuller JC, et al. Multicenter phase II trial of preoperative induction chemotherapy followed by chemoradiation with docetaxel and cisplatin for locally advanced esophageal carcinoma (SAKK 75/02). Ann Oncol. 2009;20(9):1522-1528.

\section{Publish your work in this journal}

Drug Design, Development and Therapy is an international, peerreviewed open-access journal that spans the spectrum of drug design and development through to clinical applications. Clinical outcomes, patient safety, and programs for the development and effective, safe, and sustained use of medicines are the features of the journal, which has also been accepted for indexing on PubMed Central. The manuscript management system is completely online and includes a very quick and fair peer-review system, which is all easy to use. Visit http://www.dovepress.com/testimonials.php to read real quotes from published authors.

Submit your manuscript here: http://www.dovepress.com/drug-design-development-and-therapy-journal 